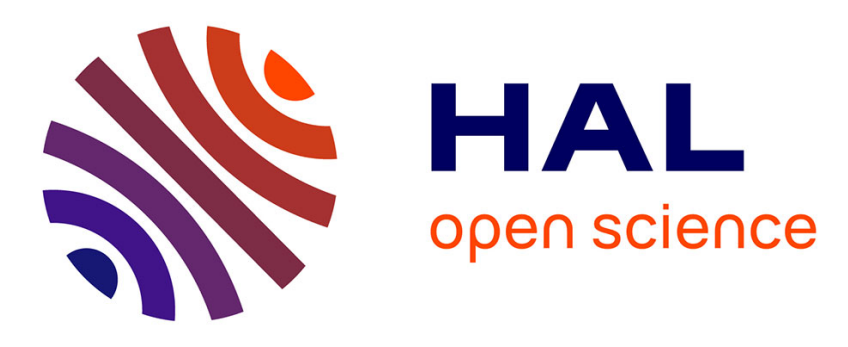

\title{
Acoustical Energy of Return Strokes: A Comparison Between a Statistical Model and Measurements
}

Arthur Lacroix, François Coulouvrat, Régis Marchiano, Thomas Farges, Jean-francois Ripoll

\section{- To cite this version:}

Arthur Lacroix, François Coulouvrat, Régis Marchiano, Thomas Farges, Jean-francois Ripoll. Acoustical Energy of Return Strokes: A Comparison Between a Statistical Model and Measurements. Geophysical Research Letters, 2019, 46 (20), 10.1029/2019GL085369 . hal-02344800

\section{HAL Id: hal-02344800 https://hal.sorbonne-universite.fr/hal-02344800}

Submitted on 25 Nov 2020

HAL is a multi-disciplinary open access archive for the deposit and dissemination of scientific research documents, whether they are published or not. The documents may come from teaching and research institutions in France or abroad, or from public or private research centers.
L'archive ouverte pluridisciplinaire HAL, est destinée au dépôt et à la diffusion de documents scientifiques de niveau recherche, publiés ou non, émanant des établissements d'enseignement et de recherche français ou étrangers, des laboratoires publics ou privés. 


\title{
Acoustical energy of return strokes: a comparison between a statistical model and measurements
}

\author{
Arthur Lacroix ${ }^{1,2}$, François Coulouvrat ${ }^{1}$, Régis Marchiano ${ }^{1}$, Thomas Farges ${ }^{2}$, \\ Jean-François Ripoll ${ }^{2}$ \\ ${ }^{1}$ Institut Jean Le Rond $\partial$ 'Alembert (UMR 7190), Sorbonne Université, 4 Place Jussieu, 75005 Paris, \\ France \\ ${ }^{2}$ CEA, DAM, DIF, F-91297 Arpajon, France
}

\section{Key Points:}

- Novel statistical model of thunder with tortuous channel and radiation-hydrodynamics simulated source.

- Good agreement found between modeled and measured acoustic energy over a distance range.

- Measurement of acoustic energy paves the way to estimate lightning deposited energy.

\section{Plain language summary}

Thunder is the remote acoustic signature of lightning. It covers a wide range of frequencies, from infrasound below $20 \mathrm{~Hz}$ to higher audible sounds. To what extent the recording of thunder can provide useful information about lightning ? As an attempt to answer this question, a new thunder model is proposed and compared with measurements made in Southern France in Fall 2012. The model relies on three key ingredients. The first one is the geometry of the lightning channel from cloud to ground, modeled as a random process whose parameters are chosen to fit well-known optical observations. The second component is the acoustical pressure wave near the discharge that originates from the hot air expanding from the lightning discharge, obtained from radiation-hydrodynamic simulations. The third aspect is propagation, assuming simply a homogeneous but sound absorbing atmosphere. Acoustic model predictions are compared at different distances with measured data with good agreements. Comparison shows, for the first time to our knowledge, that the easily measured overall acoustic energy at one distant microphone, can inform us about the order of magnitude of deposited energy within the lightning channel.

Corresponding author: Arthur Lacroix, arthur.lacroix@upmc.fr 


\begin{abstract}
This letter proposes a new statistical model of thunder. The tortuous geometry of the emitting return stroke is randomly generated to fit observations of negative cloud-to-ground discharges. Pressure waves are initialized by radiation-hydrodynamics simulations and linearly propagated into an isothermal atmosphere incorporating standardized sound absorption. The thunder pressure frequency signal is defined as the product of the input pressure governed by a deposited energy with the stochastic frequency response of the elongated discharge. We find the low frequency content of thunder is mostly due to stroke elongation originating from tortuosity. Acoustic energy per stroke length and spectrum slope are statistically compared to measurements, with good agreement found. We show both a near- and a farfield regime of the acoustical energy over distance described by two different power laws. The correlation found between the lightning energy and the acoustic energy paves the way for using thunder measurement to estimate deposited energy.
\end{abstract}

\title{
1 Introduction
}

The most advanced theory of thunder is the string-of-pearl model derived by Few $(1969,1995)$ describing the audible content of thunder. This model is based on the classical self-similar shock wave theory of Lin (1954) (see also Taylor (1950)), which was numerically extended by Brode (1955), and leads to a Kinney-type shock wave (Kinney, Graham, and Raspet (1986)) emitted by the return stroke. Few also introduced the channel tortuosity in his model. However tortuosity effects were finally neglected as stated in Ribner and Roy (1982) who particularly investigated effects of tortuosity on thunder. Using common lightning parameters (temperature, deposited energy, ...) in his simulations, Few found that the power spectrum of thunder must be sharply centered around 50-200 $\mathrm{Hz}$, which cannot explain the low frequencies observed by Holmes, Brook, Krehbiel, and McCrory (1971). Thunder infrasound is supposed to be produced by electrostatic release in the cloud during the discharge (Dessler (1973); Pasko (2009); Wilson (1921)). However, thanks to 3D acoustical reconstructions performed by Gallin et al. (2016); Lacroix, Farges, Marchiano, and Coulouvrat (2018) achieved to acoustically separate the contribution of the different parts of a discharge. They showed that most of acoustic energy, including infrasound, is produced by the return stroke. Moreover they presented several thunder spectra. None of them had a peak around a central frequency. They rather showed a roughly flat response below $100 \mathrm{~Hz}$. In order to reconcile these observations with Few's model over the whole frequency range, we propose an enhanced model of thunder. This novel model is constituted of three ingredients (i) a radiation-hydrodynamics source model, (ii) a realistic return stroke tortuosity, (iii) a propagation model including atmospheric absorption (Bass, 1980). The results of the model will be statistically compared with actual thunder measurements performed during the HyMeX SOP1 campaign (Defer et al. (2015); Gallin et al. (2016); Lacroix et al. (2018)).

\section{Statistical model}

\subsection{Temporal waveform source model}

The lightning return stroke source model originates from radiation-hydrodynamics simulations in one-dimensional cylindrical geometry (see Ripoll, Zinn, Colestock, and Jeffery (2014); Ripoll, Zinn, Jeffery, and Colestock (2014) for details). The hydrodynamics solver is based on the Von Neumann-Richtmyer shock capturing scheme, that is a Lagrangian coordinate finite difference scheme (Richtmyer and Morton (1967)) used to solve Euler equations and made to capture and follow the shock dynamics. The scheme is fully described in Zinn (1973). The approach is similar to the lightning and long spark models described in the pioneering work of Plooster (1970, 1971a, 1971b) and Paxton, Gardner, and Baker (1986). The equation of state of air is tabulated from Hilsenrath, Green, and Beckett (1957); Hilsenrath and Klien (1963). The multifrequency radiation trans- 
port is solved exactly using the discrete-ordinates method Carlson and Lapthrop (1965); Modest (2002). Chemistry and electrodynamics effects described in Ripoll, Zinn, Colestock, and Jeffery (2014), are absent from these simulations. They are simply initiated with a specified input energy per unit length $E_{0}$, at $t=0$ within a specified channel radius, $R_{0}$, located at an altitude $H_{0}$ as done in Ripoll, Zinn, Colestock, and Jeffery (2014) (see their Table 1). Three cases of, respectively, $\left(E_{0}=4 \mathrm{~J} / \mathrm{cm}, R_{0}=1.5 \mathrm{~mm}\right),\left(E_{0}=\right.$ $\left.28 \mathrm{~J} / \mathrm{cm}, R_{0}=1.5 \mathrm{~mm}\right),\left(E_{0}=60 \mathrm{~J} / \mathrm{cm}, R_{0}=1 \mathrm{~cm}\right)$ at $H_{0}=8 \mathrm{~km}$ (i.e. with an ambient density of $\left.\rho_{0}=5.41 \times 10^{4} \mathrm{~g} / \mathrm{cm}^{3}\right)$ have been chosen to cover the energy spectrum of common return strokes (Borovsky (1998); Cooray (2003)). We use 360 nodes in the radial direction so that the smallest cell can reach $10^{-2} \mathrm{~cm}$ in the shock layer thanks to the Lagrangian method.

Figure 1A displays the evolution of the pressure represented versus distance at different times for the case $60 \mathrm{~J} / \mathrm{cm}$. The initial hot air channel generates a shock and expands. Zoom on Figure 1A shows that the shock is well captured during the whole simulation ending at $30 \mathrm{~ms}$. The shock is located at the peak pressure and a rarefaction zone occurs behind it. Rarefaction waves of small amplitude moves backward in the direction of the origin, reflects there, moves then outward, faster that the main shock until they reach it (Brode (1955); McFadden (1952); Plooster (1970)). The maximal temperature is here $T_{0} \approx 10,000 \mathrm{~K}$ at $t=0$ (Figure $1 \mathrm{~B}$ ), a moderate value due to the initial radius $\left(R_{0}=1 \mathrm{~cm}\right)$. Radiation escapes immediately and continuously the hot region during the channel expansion since the temperature is always lower than $\approx 15,000 \mathrm{~K}$ (Ripoll, Zinn, Jeffery, and Colestock (2014)). Radiation cooling and some absorption ahead the shock contributes to create a complex profile of the hot front. At large times $(t=1,10 \mathrm{~ms})$, the shock becomes also visible through a temperature peak of a few Kelvin (Figure 1B') that progressively separates from the main hot cooling plasma channel located behind the shock and which has stopped expanding. At $10 \mathrm{~ms}$ this wave has clearly separated and appears as an acoustical oscillation around the ambient state for both temperature and pressure. Cooling in the hot now static region $(R<7 \mathrm{~cm})$ should be faster due to turbulent convection from the surrounding cool air that penetrates the hot channel but is not accounted for in the model. This is assumed not to affect our study that focuses on the dynamic signature of the acoustical shock overpressure.

Figure 1C shows the normalized overpressure defined as $\left(P(r, t)-P_{0}\right) / \sqrt{r / r_{r e f}}$ (with $P_{0}$ the ambient pressure and $r_{\text {ref }}$ such that the maximum value is 1 ) evolution at different distances $([5-600] \mathrm{cm}$ ) during $28 \mathrm{~ms}$ every $2 \mu \mathrm{s}$. For each location, one observes (1) a pressure increase as the peak approaches, then (2) the peak, and (3) the depressurization of the rarefaction wave behind it giving a negative value. At the earliest time, close to the source $(R<40 \mathrm{~cm}$, zoom 1C'), the peak phase (1) has a ten times greater amplitude than the depressurization phase (3) and the temporal waveform is very close to a Kinney wave (as theoretically expected by Few (1969) and measured by Karzova et al. (2015)). However during its propagation, the waveform significantly changes. The shock, initially in compression, gradually becomes a relaxation one and the positive phase (1) decreases whereas the negative one (3) increases. Finally, when the shock has fully separated from the hot channel (i.e. $R \geq 200 \mathrm{~cm}$ ), both phases (1) and (3) balance each other to give an almost anti-symmetric pressure profile. For these reasons we consider the wave as having reached the acoustic regime at this distance. The pressure wave profile at $200 \mathrm{~cm}$ of the channel is now chosen as the source term of this thunder model. Figure 1D illustrates the pressure profiles for the 3 deposited energies, showing that once the acoustic regime is reached, the deposited energy only changes the amplitude of the temporal waveform. Note that the N-wave model and Kinney wave model, both simpler alternative source models, do not have the symmetric shape of the present source model (once the acoustical regime has been reached), itself retained since recorded pressure signals are indeed found to be symmetric at far distance from the source (Lacroix, $\mathrm{PhD}$ ). Figure 1E shows the spectra of the three cases, as in Few (1969), a peak frequency 
at $150 \mathrm{~Hz}$ is observed. A sharp decay above the peak and a long tail down to the infrasonic range are visible.

\subsection{Random generation of realistic return strokes}

Following Ribner and Roy (1982), we developed a 3D lightning geometry generator based on the stereophotographic observations of natural lightning strokes by Hill (1968) for negative Cloud-to-Ground discharges (-CG) that represent about $90 \%$ of the total number of CGs. The lightening stroke is divided into steps of length $h_{i}$ (here $h_{i}$ is assumed to be constant equal to $8 \mathrm{~m}$ in agreement with LeVine and Gilson (1984) and Glassner (2000)). The deflection angle between two different steps follows a Gaussian distribution with a mean absolute value of $16.3^{\circ}$. Note that this angle does not seem to depend on the step length. Starting from an initial point $\left(x_{0}, y_{0}, z_{0}\right)$, the extremities of the steps $\left(x_{i}, y_{i}, z_{i}\right)$ are computed successively:

$$
\left\{\begin{array}{l}
x_{i+1}=x_{i}+h_{i}\left(\cos \theta_{i}^{\prime} \sin \Theta_{i} \cos \Psi_{i}+\sin \theta_{i}^{\prime} \cos \varphi_{i}^{\prime} \cos \Theta_{i} \cos \Psi_{i}-\sin \theta_{i}^{\prime} \sin \varphi_{i}^{\prime} \sin \Psi_{i}\right) \\
y_{i+1}=y_{i}+h_{i}\left(\cos \theta_{i}^{\prime} \sin \Theta_{i} \sin \Psi_{i}+\sin \theta_{i}^{\prime} \cos \varphi_{i}^{\prime} \cos \Theta_{i} \sin \Psi_{i}+\sin \theta_{i}^{\prime} \sin \varphi_{i}^{\prime} \cos \Psi_{i}\right) \\
z_{i+1}=z_{i}+h_{i}\left(\sin \theta_{i}^{\prime} \cos \varphi_{i}^{\prime} \sin \Theta_{i}-\cos \theta_{i}^{\prime} \cos \Theta_{i}\right) .
\end{array}\right.
$$

The spherical angles $\Theta_{i}$ (relative to the vertical direction $z$ ) and $\Psi_{i}$ (in the horizontal plane $(x, y))$ denote the orientation of the $\mathrm{i}^{\text {th }}$ step. Angles $\theta_{i}^{\prime}$ and $\varphi_{i}^{\prime}$ measure the deflection of the new step $i+1$ relative to the previous one $i$. The $\theta_{i}^{\prime}$ angle determines the aperture of the cone of height $h_{i}$ swept by the $i+1$ extremity. The $\varphi_{i}^{\prime}$ angle gives the position of this extremity around the base of the latter cone. These two angles are randomly calculated thanks to the two following probability density functions (pdf):

$$
\begin{gathered}
p\left(\theta_{i}^{\prime}\right)=a \exp \left(-\frac{\left(\theta^{\prime}+\bar{\Theta}_{i} / N_{\theta}\right)^{2}}{\Delta \theta^{2}}\right) \\
q\left(\varphi_{i}^{\prime}\right)=\frac{a}{2} \exp \left(-\frac{\left(\varphi^{\prime}-\bar{\Psi}_{i} / N_{\varphi}\right)^{2}}{\Delta \varphi^{2}}\right)+\frac{a}{2} \exp \left(-\frac{\left(\varphi^{\prime}+\bar{\Psi}_{i} / N_{\varphi}\right)^{2}}{\Delta \varphi^{2}}\right) .
\end{gathered}
$$

Here $a$ is a normalization term, $\bar{\Theta}_{i}$ is the average of the angles $\theta_{i}^{\prime}$ on the $k_{\theta}$ previous segments, $k_{\theta}$ is the so-called memory term (see Ribner and Roy (1982) equation (10)). $N_{\theta}$ is a bias term and $\Delta \theta$ is the standard deviation chosen to recover Hill's statistical observations. Ribner and Roy (1982) proposed a uniform pdf between 0 and $2 \pi$ for the angle $\varphi_{i}^{\prime}$. Here we instead propose equation (3). It corresponds to a double Gaussian function reflecting the fact that the $\varphi_{i}$ angle has the same occurrence probability as its opposite. The chosen form for $q\left(\varphi_{i}^{\prime}\right)$ is, similarly to $p\left(\theta_{i}^{\prime}\right)$, biased thanks to the parameter $N_{\varphi}$ and centered around a memory term, $\bar{\Psi}_{i}$, which is the average of the $k_{\varphi}$ previous $\Psi$ angles. Compared with the Ribner and Roy (1982) model, this additional pdf induces a smoother shape of the lightning through memory effects on both angles.

In order to validate the process, 10,000 strokes have been generated with parameters $\left(x_{0}=0 m ; y_{0}=0 m ; z_{0}=5000 m ; \Delta \theta=27.08^{\circ}, N_{\theta}=20, k_{\theta}=4, \Delta \varphi=45.0^{\circ}\right.$, $N_{\varphi}=20$ and $\left.k_{\varphi}=100\right)$. The altitude of $5000 \mathrm{~m}$ is the typical initiation height for negative CG, inside the cloud lower layer of negative electric charges (Rakov (2013)). Figure $2 \mathrm{~A}$ shows three examples of randomly generated lightning strokes. Their aspect is quite realistic, with fine geometrical structures including multiple changes of direction. Figure $2 \mathrm{~B}$ plots the mean absolute value of the deflection angles between two successive steps along each simulated stroke in the two vertical planes $(x, z)$ and $(y, z)$. As expected similar Gaussian distributions are recovered. The mean value of $16.3^{\circ}$ of Hill (1968) statis- 
tics is obtained by adjusting the value of $\Delta \theta$. Figure $2 \mathrm{C}$ shows the statistical distribution of the total length of the 10,000 generated tortuous strokes. The distribution appears to be Poisson-like with a peak value of about $8 \mathrm{~km}$ in agreement with literature data (see Rakov (2013)).

\subsection{Propagation}

Each step of the tortuous stroke is sampled with 80 equispaced point sources (so as to capture frequencies up to $6000 \mathrm{~Hz}$ ). Each source emits the same signal (see subsection 2.1) at the same time. The formation time of the lightning (typically $0.1 \mathrm{~ms}$ ) is much shorter than the acoustic wave propagation time (typically $3 \mathrm{~s}$ at $1 \mathrm{~km}$ ). Moreover, the temperature along the channel does not vary significantly due to the weak effect of the change of the ambient density with elevation (Ripoll, Zinn, Jeffery, and Colestock (2014)). Assuming a linear propagation, the signal received at a distance $d$ from a point source emitting a signal $s(t)$ is simply: $p(t)=G(d, t) * s(t)$ where $*$ denotes the convolution product and $G(d, t)$ the Green function between the source and the observer. So, the signal received by an observer from the whole lightning is the sum of all individual contributions:

$$
P_{\text {tot }}(t)=\sum_{n=1}^{N} G_{n}\left(d_{n}, t\right) * s(t)=G_{t o t}(t) * s(t),
$$

where $d_{n}$ is the distance between the $n^{\text {th }}$ source and the observer. The sum of all Green functions, $G_{t o t}$, is the impulse response of the overall lightning stroke. We assume a straight line propagation in a homogeneous medium at constant sound speed $c_{0}=340 \mathrm{~m} / \mathrm{s}$ supported by Gallin et al. (2016) and Lacroix et al. (2018) who presented 3D acoustic reconstructions of lightning discharges that compare favorably with very high frequency electromagnetic reconstructions. Moreover, the AROME-WMED meteorology model, which was available during the HyMeX SOP1 campaign, provided outputs with a horizontal scale of $1 \mathrm{~km}$ and a time scale of 1 hour (Defer et al. (2015)). This is far too insufficient to match the source scale of $8 m$ used here. By using the free field Green function, equation (4) becomes in the frequency domain:

$$
\tilde{P}_{t o t}(f)=\tilde{G}_{t o t}(f) \times \tilde{s}(f) \text { with } \tilde{G}_{t o t}(f)=\sum_{n=1}^{N} \frac{e^{-j k_{0} d_{n}}}{4 \pi d_{n}}
$$

with $k_{0}=2 \pi f / c_{0}$ the wave number and $\tilde{G}_{t o t}(f)$ the frequency response of the stroke. This formulation enables us to separate the contribution of the temporal shape of the source (subsection 2.1) from the influence of both geometry and the propagation. However, Bass (1980) has shown that atmospheric absorption should be taken into account to properly model thunder. We use the ISO-9631 standard and modify the wave number expression by: $k_{a b s}(f)=k_{0}[1+\nu(f)-j \alpha(f)]$, where $\nu(f)$ measures the wave dispersion and $\alpha(f)$ its attenuation. These two effects are linked to Nitrogen and Oxygen molecular relaxation. The value of these functions is standardized according to pressure, temperature and relative humidity. More details are available in ISO-9613-1 (1993). Then, operator $\tilde{G}_{\text {tot }}$ becomes:

$$
\tilde{G}_{t o t}(f)=\sum_{n=1}^{N} \frac{e^{-2 \pi f \tau_{n} \alpha(f)}}{4 \pi d_{n}} e^{-2 j \pi \tau_{n} f[1+\nu(f)]},
$$

where $\tau_{n}=d_{n} / c_{0}$ is the time of flight between the $\mathrm{n}^{\text {th }}$ source and the observer. To compare with Few's model (equation (11)), we calculate the mean square of the received spectrum: 


$$
\left|\tilde{P}_{t o t}(f)\right|^{2}=\frac{|\tilde{s}(f)|^{2}}{16 \pi^{2}}\left(\sum_{n=1}^{N} \frac{e^{-4 \pi f \alpha(f) \tau_{n}}}{d_{n}^{2}}+\sum_{\substack{n, m=1 \\ n \neq m}}^{N} \frac{e^{-2 \pi f \alpha(f)\left(\tau_{n}+\tau_{m}\right)}}{d_{n} d_{m}} \cos \left(2 \pi f\left[\tau_{n}-\tau_{m}\right][1+\nu(f)]\right)\right)
$$

For an observer over a rigid ground, this result has to be quadrupled. The first difference with Few's model is the additional terms due to absorption $(\alpha(f))$ and dispersion $(\nu(f))$. The first sum can be understood as a purely geometrical term remaining even if all the waves arrive without any phase difference. The second sum takes into account all phase shifts due to the travel time differences between the sources and the observer. Equation (11) of Few (1969) includes a similar term (without absorption) but overlooked it in practice. This may be true at high frequencies because of destructive interferences, but not at low frequencies. This term is therefore fully taken into account.

\subsection{Model analysis}

Figure 3 shows the resulting frequency responses $\left|\tilde{G}_{\text {tot }}(f)\right|$ (left column) and the signal spectra $\left|\tilde{P}_{t o t}(f)\right|$ (right column) simulated at four distances from the impact point for the three randomly generated geometries of Figure $2 \mathrm{~A}$ and for a deposited energy $E_{0}=$ $60 \mathrm{~J} / \mathrm{cm}$ calculated at $200 \mathrm{~cm}$. Each case is compared with a $5 \mathrm{~km}$ height straight vertical stroke (plotted in dashed lines), for which the frequency response is a Hankel function of the first kind, $H_{0}^{1}\left(k_{a b s} r\right)$, the analytical solution for a cylindrical wave. This one is normalized to each random simulation using $\left|\tilde{G}_{t o t}(f=300 \mathrm{~Hz})\right|$ at $12,800 \mathrm{~m}$.

Looking at the frequency responses, we first observe that they all globally follow the decreasing behavior expected from the vertical stroke. This proves the extended geometry favors infrasound emergence, whatever the return stroke tortuosity is. This corroborates field observations of Lacroix et al. (2018) showing an important low frequency content of return strokes. Also observed is the increase with distance of the negative slopes of all frequency responses, whatever the geometry is. This evolution is clearly due to atmospheric absorption. Neglecting absorption would lead to an artificially flat frequency response (Bass (1980)). Beyond these common trends, significant variability from stroke to stroke are visible at short distances. This is explained by the channel individual tortuosity inducing random constructive or destructive interferences, and leads to quite different individual fluctuations in the extreme near field $(100 \mathrm{~m})$. For instance, strokes 1 and 3 show frequency response mean levels centered around the case of a straight vertical channel. However they exhibit two different kinds of modulation: a slow modulation $(\approx 100 \mathrm{~Hz}$ width) for stroke 1 , and a more rapid one ( $\approx 10 \mathrm{~Hz}$ width) for stroke 3 . On the contrary, stroke 2 shows less fluctuations around a mean value that is one order of magnitude lower than the straight lightning. This may correspond to a case where destructive interferences are dominant. At 1,600 $\mathrm{m}$, the large modulations of stroke 1 have almost disappeared, while those of stroke 3 are reduced in amplitude and stroke 2 now approaches the mean straight lightning case. Thus we can observe a near- to farfield transition for which the nearfield variability (and the significant deviations from the ideal straight lightning) due to channel random tortuosity progressively dampens with distance. The positioning of this transition will be quantified more precisely in the following section.

Resulting spectra are the product of the stochastic frequency response with the deterministic source spectrum. As the source presents an emission peak around $150 \mathrm{~Hz}$, and as the frequency response tends to favor low frequencies below $50 \mathrm{~Hz}$, the combination of both antagonistic effects leads to a relatively flat spectrum in the nearfield, between roughly 1 and $200 \mathrm{~Hz}$. Beyond $200 \mathrm{~Hz}$ the slope is decreasing with frequency as a signature of the source. All nearfield fluctuations of the frequency responses are con- 
veyed in the spectra. In the farfield, fluctuations dampen in amplitude and the influence of tortuosity fades away while absorption becomes determinant. These characteristics are globally in good agreement with observations of Lacroix et al. (2018) (Figure 10). For instance, at short distances, relatively flat spectra are observed, with significant fluctuations in some cases (see for instance event $2($ at $380 \mathrm{~m}$ ) in Figure $8 \mathrm{C}$ in Lacroix et al. (2018)). In the farfield, lower variability is observed as well as an increasing negative spectrum slope with distance. This good qualitative agreement gives us confidence in this new thunder model.

\section{Statistical comparison with thunder measurements}

A database of 36 measured return strokes during three different storms (Lacroix et al. (2018)) is compared with 72 generated strokes at 9 different distances (from 100 to $25,600 \mathrm{~m}$ ). These 72 events are selected among the 10,000 cases to get an isotropic distribution of the impact point relatively to a fixed emission point. Figure $4 \mathrm{~A}$ shows the acoustical energy per stroke length $E_{l}=E_{a c} / L$ as function of the impact point distance $r$, where $E_{a c}$ is the acoustic energy received at measurement points over the signal duration (as defined in equation (3) of Lacroix et al. (2018)), and $L$ is the stroke channel length (see figure 2C). For measured data, $L$ is estimated from acoustical reconstruction. Both simulated and reconstructed strokes have a mean length value of about $8 \mathrm{~km}$. At each distance, we represent the mean value of the 72 numerically computed $E_{l}$ energies, with their standard deviations. Changing $E_{0}$ modifies only the wave amplitude in the acoustical regime, not its spectral content (see section 2.1). We also plot a power law fit of the $28 \mathrm{~J} / \mathrm{cm}$ case. Between 100 and 3, $600 \mathrm{~m}$ the power follows the $r^{-1}$ cylindrical wave decay and the $r^{-2}$ spherical wave decay beyond 3,600 and 25,600 $\mathrm{m}$. This change of slope confirms statistically the existence of a near- and a farfield behavior already discussed in section 2.4. This difference between near- and farfield is also clearly visible in the strong reduction of variability; from 2 orders of magnitude at $100 \mathrm{~m}$ to only a factor about 2 at 12,800 $\mathrm{m}$. As found in section 2.4, the tortuosity of the lightning stroke induces a strong variability but mostly below $1 \mathrm{~km}$. Measurements of $E_{l}$ obtained during the HyMeX SOP1 campaign (Defer et al. (2015); Gallin et al. (2016); Lacroix et al. (2018)) are also represented. We also indicate the ambient noise level one hour before the most intense thunderstorm (October 26th). Note that compared with Lacroix et al. (2018) a low pass filter above $0.5 \mathrm{~Hz}$ is added to remove the significant swell contribution. The most striking feature of this comparison is the good agreement between numerical and experimental acoustic energy values, especially between 1,000 and 10,000 $\mathrm{m}$. All experimental data of -CG fit the deposited energy range [4-60] $\mathrm{J} / \mathrm{cm}$. The amount of deposited energy within a stroke is still subject to debate, either in the range $[0-1,000] \mathrm{J} / \mathrm{cm}$ according to Cooray (2003) or in the range [0-100] $\mathrm{J} / \mathrm{cm}$ according to Borovsky (1998). Our results are in better agreement with this last reduced range. To our knowledge this is the first time that deposited energy is estimated thanks to a distant acoustical measurement. In the nearfield $(<1 \mathrm{~km})$ and although the two experimental points agree with the model, more data within that range would be necessary. For distant observers $(>10 \mathrm{~km})$, the four -CG points are within the noise level. Concerning the $10+\mathrm{CG}$ measurements, our tortuosity model is not adapted for them, but distant observations (> $5 \mathrm{~km}$ ) probably minimize tortuosity influence. Six of them are within our $E_{0}$ energy range, two are above ( $E_{0}$ is generally expected to be higher for +CG than for -CG (Rakov (2013))), and two are below the noise level. There seems to be more variability of $+\mathrm{CG}$ energies than for -CG, but this would need to be confirmed with more data. Finally, note that Lacroix et al. (2018) had a global "intermediate" experimental power fit with distance as $r^{-1.5}$. One of their hypothesis for this behavior was the role of non-linearity during propagation. Here it clearly results from the linear near- to farfield transition due to the stroke geometry. 
Figure 4B displays the evolution of the spectra slope for both experimental and numerical (independent of $E_{0}$ ) data. The experimental decreasing behavior with distance is numerically recovered. Moreover some positive slopes are observed with similar proportions only in the nearfield: 5 of 36 experimental cases (about 13\%), and 50 of $648 \mathrm{nu}-$ merical cases (about 8\%). A linear regression of the mean numerical data matches well the measurements (Lacroix et al. (2018)). Our model simulates therefore the right order of magnitude of the spectrum slope and its evolution with distance. However it underestimates its variability. Meteorological spatial and temporal inhomogeneities (mostly wind and temperature gradients) could be a possible explanation of this underestimation.

\section{Conclusion}

This letter presents a new thunder model based on three distinct components: (i) a radiation-hydrodynamics source model, (ii) a random lightning geometry generator adapted to -CG, (iii) a propagation model including absorption. Model shows tortuosity is at the origin of a nearfield regime $(<3,600 \mathrm{~m})$ in which acoustic energy variability is very high, up to two orders of magnitude. On the contrary, in the farfield, variability fades away due to absorption. The mean behavior of a tortuous lightning stroke is the one of a rectilinear stroke. Consequently, the important low frequency content of thunder is mostly due to stroke elongation. Acoustic energy per stroke length, $E_{l}$, and spectrum slope were statistically compared with measurements. The good agreement for both quantities proves all three model ingredients are required to correctly model thunder. More specifically near- and farfield behaviors were recovered for the $E_{l}$ evolution over distance with two different power laws (cylindrical versus spherical divergence). Finally an important result is the correlation between the deposited energy at the source and the received acoustic energy per stroke length. The explored range [4-60] J/ cm for deposited energy, in compliance with Borovsky (1998), predicts measured acoustic thunder energies in close agreement with measurements. Such a correlation is here obtained for the first time to our knowledge. This result opens new insight about a potential link between acoustic and electric lightning quantities.

\section{Acknowledgments}

The authors acknowledge the HyMeX program for supplying the data, sponsored by grants MISTRALS/HyMeX and ANR-11-BS56-0005 IODA-MED project. Present results have been obtained within the frame of the LETMA (Laboratoire ETudes et Modélisation Acoustique), Contractual Research Laboratory between CEA, CNRS, Ecole Centrale Lyon, C-Innov, and Sorbonne Université. The acoustic signals are available on the HyMeX database (http://mistrals.sedoo.fr/HyMeX/).

\section{References}

Bass, H. E. (1980). The propagation of thunder through the atmosphere. The Journal of the Acoustical Society of America, 67(6), 1959-1966. doi: 10.1121/1 .384354

Borovsky, J. E. (1998). Lightning energetics: Estimates of energy dissipation in channels, channel radii, and channel-heating risetimes. Journal of Geophysical Research: Atmospheres, 103(D10), 11537-11553. doi: 10.1029/97JD03230

Brode, H. L. (1955). Numerical solutions of spherical blast waves. Journal of Applied Physics, 26(6), 766-775. doi: 10.1063/1.1722085

Carlson, B. G., \& Lapthrop, K. D. (1965). Transport theory, the method of discrete ordinates (LA-3251-MS, Ed.). LANL Rep.

Cooray, V. (2003). The Lightning Flash (T. I. of Electrical Engineers, Ed.).

Defer, E., Pinty, J.-P., Coquillat, S., Martin, J.-M., Prieur, S., Soula, S., .. Molinié, 
G. $(2015,02)$. An overview of the lightning and atmospheric electricity observations collected in Southern France during the HYdrological cycle in Mediterranean EXperiment (HyMeX), Special Observation Period 1. Atmospheric Measurement Techniques, 8, 649-669.

Dessler, A. J. (1973). Infrasonic thunder. Journal of Geophysical Research, 78(12), 1889-1896. Retrieved from http://dx.doi.org/10.1029/JC078i012p01889 doi: $10.1029 /$ JC078i012p01889

Few, A. A. (1969). Power spectrum of thunder. Journal of Geophysical Research.

Few, A. A. (1995). Acoustic radiation from lightning. In H. Volland (Ed.), Handbook of atmospheric electrodynamics - volume ii (pp. 1-31). CRC Press.

Gallin, L.-J., Farges, T., Marchiano, R., Coulouvrat, F., Defer, E., Rison, W., ... Nuret, M. (2016). Statistical analysis of storm electrical discharges reconstituted from a lightning mapping system, a lightning location system, and an acoustic array. Journal of Geophysical Research: Atmospheres, 121(8), 3929-3953. doi: 10.1002/2015JD023745

Glassner, A. (2000, Mar). The digital ceraunoscope: synthetic thunder and lightning. i. IEEE Computer Graphics and Applications, 20(2), 89-93. doi: 10 $.1109 / 38.824552$

Hill, R. D. (1968). Analysis of irregular paths of lightning channels. Journal of Geophysical Research, 73, 1897-1906.

Hilsenrath, J., Green, S., \& Beckett, C. W. (1957). Thermodynamic properties of highly ionized air (Tech. Rep.). Air Force Special Weapons Center, AFSWCTR-56-35

Hilsenrath, J., \& Klien, M. (1963). Tables of thermodynamic properties of air in chemical equilibrium including second virial corrections from $1500 \mathrm{~K}$ to 15000 $K$ (Tech. Rep.). Arnold Engineering Development Center, Air Force Systems Command, AEDC-TDR-63-161.

Holmes, C. R., Brook, M., Krehbiel, P., \& McCrory, R. (1971). On the power spectrum and mechanism of thunder. Journal of Geophysical Research, 76 (9), 2106-2115. doi: 10.1029/JC076i009p02106

ISO-9613-1. (1993). Acoustics - Attenuation of sound during propagation outdoors Part 1: Calculation of the absorption of sound by the atmosphere (Tech. Rep.). Geneva: ISO Technical committee.

Karzova, M. M., Yuldashev, P. V., Khokhlova, V. A., Ollivier, S., Salze, E., \& Blanc-Benon, P. (2015). Characterization of spark-generated N-waves in air using an optical Schlieren method. The Journal of the Acoustical Society of America, 137(6), 3244-3252. doi: 10.1121/1.4921026

Kinney, G. F., Graham, K. J., \& Raspet, R. (1986). Explosive shocks in air. Springer Science \& Business Media. Retrieved from https://doi.org/ 10.1121/1.394030 doi: 10.1121/1.394030

Lacroix, A., Farges, T., Marchiano, R., \& Coulouvrat, F. (2018). Acoustical measurement of natural lightning flashes: reconstructions and statistical analysis of energy spectra. Journal of Geophysical Research - Atmosphere, 123, 1204012065 .

LeVine, D. M., \& Gilson, B. (1984, May). Tortuosity of lightning return stroke channels (Tech Report). Greenbelt, Maryland: Goddard Space Flight Center, Nationale Aeronautics and Space Administration (NASA).

Lin, S. C. (1954). Cylindrical shock waves produced by an instantaneous energy release. Journal of Applied Physics, 25(1), 54-57. doi: http://dx.doi.org/10 $.1063 / 1.1721520$

McFadden, J. A. (1952). Initial behavior of a spherical blast. Journal of Applied Physics, 23(11), 1269-1275. doi: 10.1063/1.1702047

Modest, M. (2002). Radiative heat transfer 2nd edition (M. Modest, Ed.). Academic Press.

Pasko, V. P. (2009). Mechanism of lightning-associated infrasonic pulses from thun- 
derclouds. Journal of Geophysical Research: Atmospheres, 114(D8), D08205.1D08205.10. (D08205) doi: 10.1029/2008JD011145

Paxton, A. H., Gardner, R. L., \& Baker, L. (1986). Lightning return stroke. A numerical calculation of the optical radiation. The Physics of Fluids, 29(8), 2736-2741. doi: 10.1063/1.865514

Plooster, M. N. (1970). Shock waves from line sources. Numerical solutions and experimental measurements. The physics of fluids, 13(11), 2665-2675.

Plooster, M. N. (1971a). Numerical model of the return stroke of the lightning discharge. Physics of Fluids, 14(10), 2124-2133. doi: http://dx.doi.org/10.1063/ 1.1693303

Plooster, M. N. (1971b). Numerical simulation of spark discharges in air. The Physics of Fluids, 14(10), 2111-2123. doi: 10.1063/1.1693302

Rakov, V. (2013, 04). The physics of lightning. Surveys in Geophysics, 34(6), 701729 .

Ribner, H. S., \& Roy, D. (1982). Acoustics of thunder : A quasilinear model for tortuous lightning. The Journal of the Acoustical Society of America, 72(6), 19111925. doi: http://dx.doi.org/10.1121/1.388621

Richtmyer, R. D., \& Morton, K. (1967). Difference Methods For Initial-Value Problems. Interscience.

Ripoll, J.-F., Zinn, J., Colestock, P. L., \& Jeffery, C. A. (2014). On the dynamics of hot air plasmas related to lightning discharges: 2. Electrodynamics. Journal of Geophysical Research: Atmospheres, 119(15), 9218-9235. (2013JD020068) doi: 10.1002/2013JD020068

Ripoll, J.-F., Zinn, J., Jeffery, C. A., \& Colestock, P. L. (2014). On the dynamics of hot air plasmas related to lightning discharges: 1. Gas dynamics. Journal of Geophysical Research: Atmospheres, 119(15), 9196-9217. (2013JD020067) doi: 10.1002/2013JD020067

Taylor, G. (1950). The formation of a blast wave by a very intense explosion I. Theoretical discussion. Proceedings of the Royal Society of London A: Mathematical, Physical and Engineering Sciences, 201(1065), 159-174. doi: 10.1098/rspa .1950 .0049

Wilson, C. T. R. (1921). Investigations on lightning discharges and on the electric field of thunderstorms. Philosophical Transactions of the Royal Society of London Series A, 221, 73-115. doi: 10.1098/rsta.1921.0003

Zinn, J. (1973). A finite difference scheme for time-dependent spherical radiation hydrodynamics problems. Journal of Computational Physics, 13(4), 569 - 590. doi: https://doi.org/10.1016/0021-9991(73)90034-X 

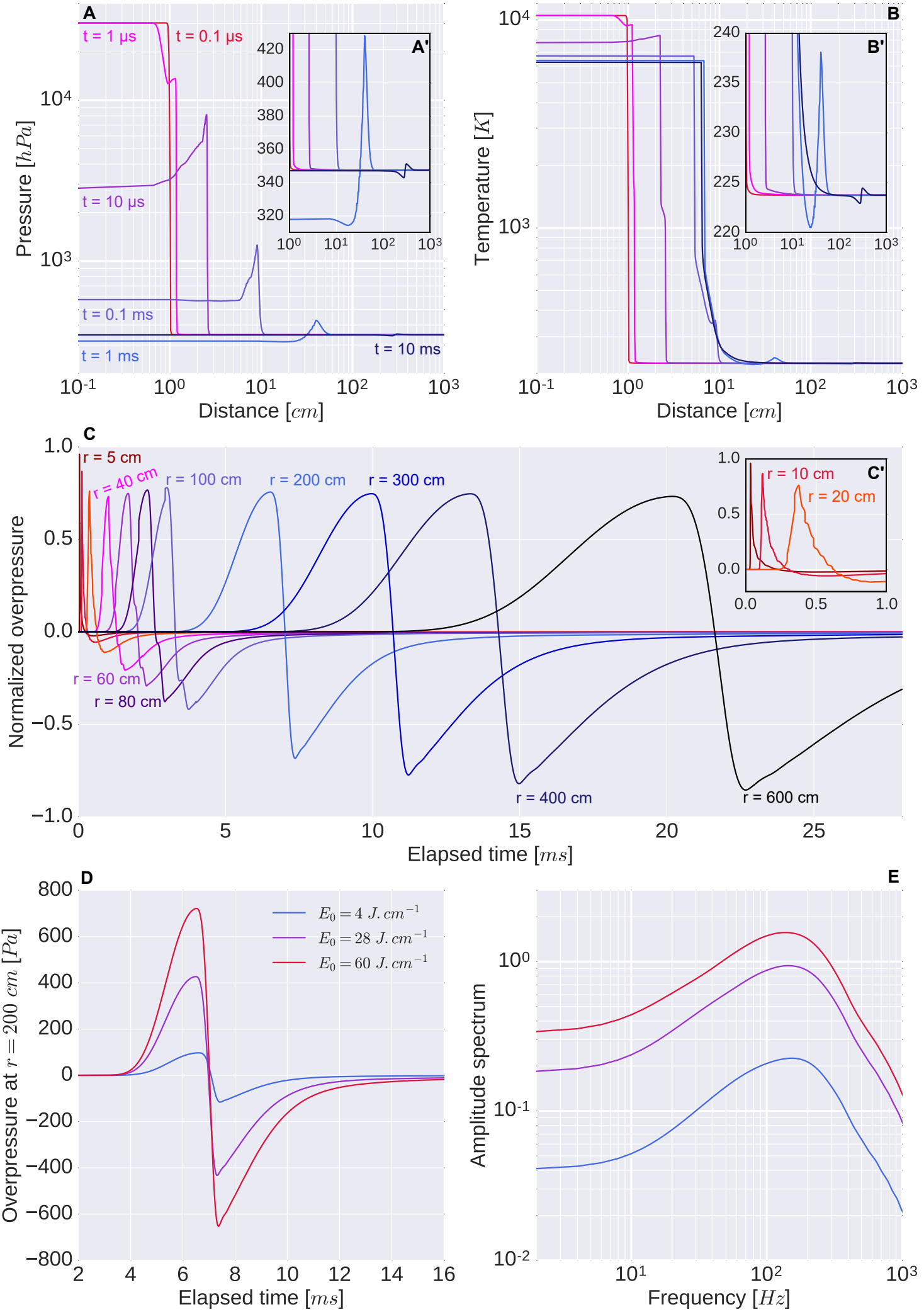

Figure 1. Evolution of the air (A) pressure and (B) temperature versus radius at six different times (from $t=10^{-7}$ to $10^{-2} \mathrm{~s}$ ) for $E_{0}=60 \mathrm{~J} / \mathrm{cm}$. Zoom of the (A') pressure and (B') temperature at $t=1$ and $10 \mathrm{~ms}$. (C) Normalized pressure versus time at different distances (from 5 to $600 \mathrm{~cm}$ ) with (C') zoom of the first three waveforms $(r=5,10,20 \mathrm{~cm})$. (D) Overpressure versus time at $200 \mathrm{~cm}$ for three different deposited energies and (E) associated spectra with same color code. 

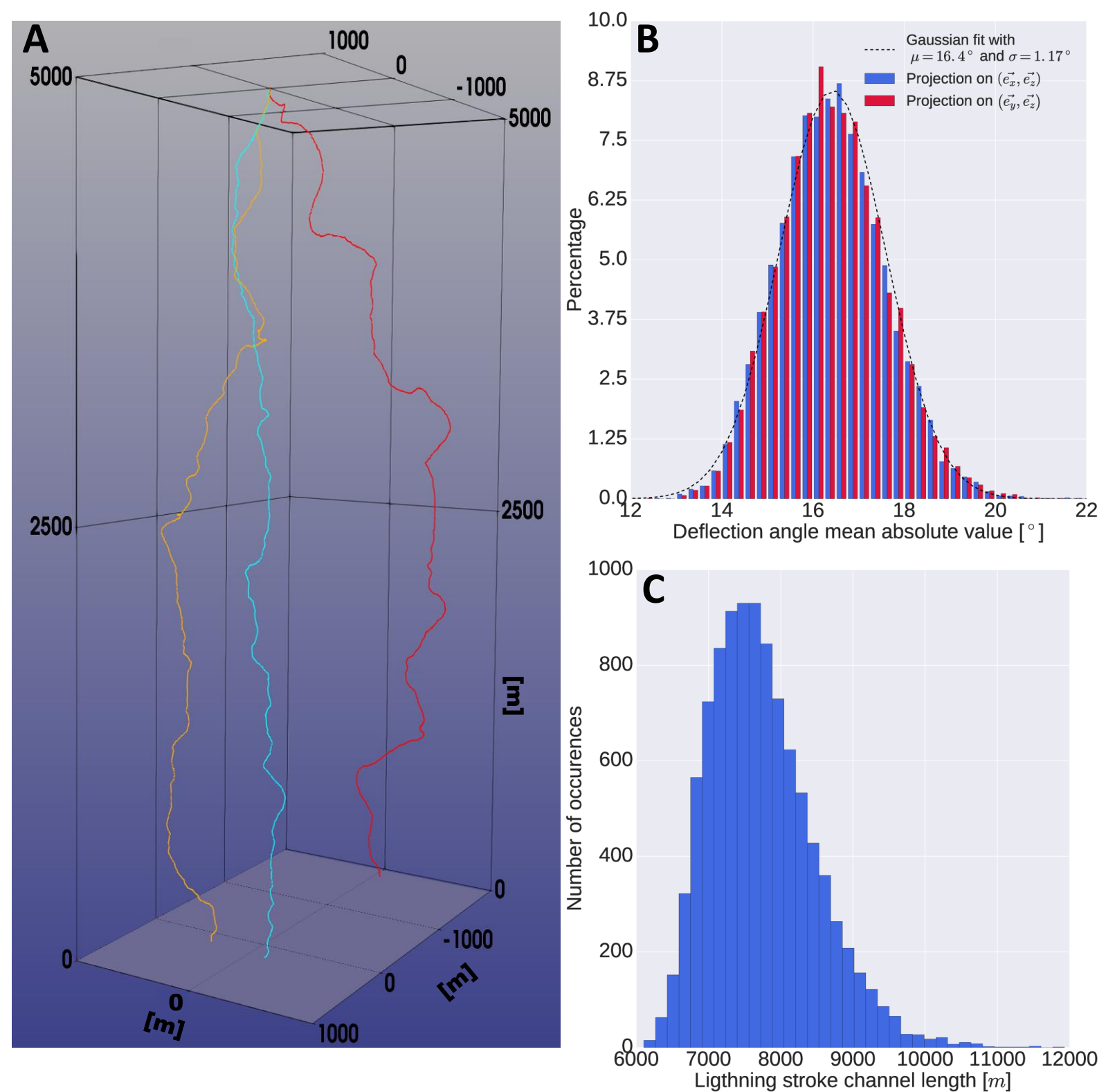

Figure 2. (A) 3 randomly generated return strokes referred as stroke 1 (blue), 2 (red) and 3 (orange). Histograms of (B) the mean absolute deflection angle and (C) the total length for 10, 000 randomly generated strokes. 

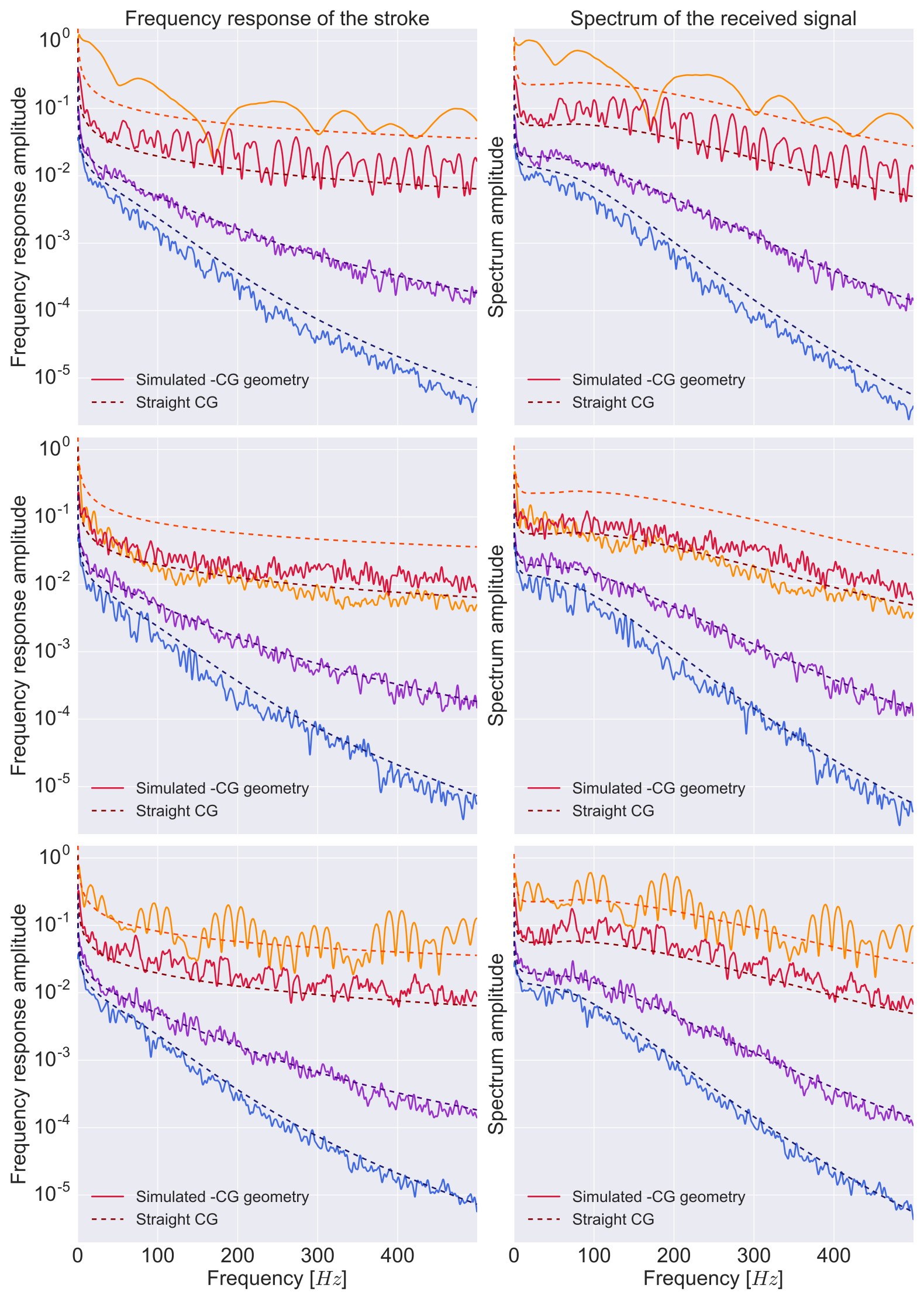

Figure 3. Left column: frequency response of the three lightning strokes (from top to bottom) showed in figure $2 \mathrm{~A}$ (solid lines) and a straight linear stroke (dashed lines) at four different distances (yellow: $100 \mathrm{~m}$, red: 1,600 $\mathrm{m}$, purple: 12,800 $\mathrm{m}$, blue: 25,600 $\mathrm{m}$ ). Right column: associated spectra of the same 3 strokes with the same color code. 

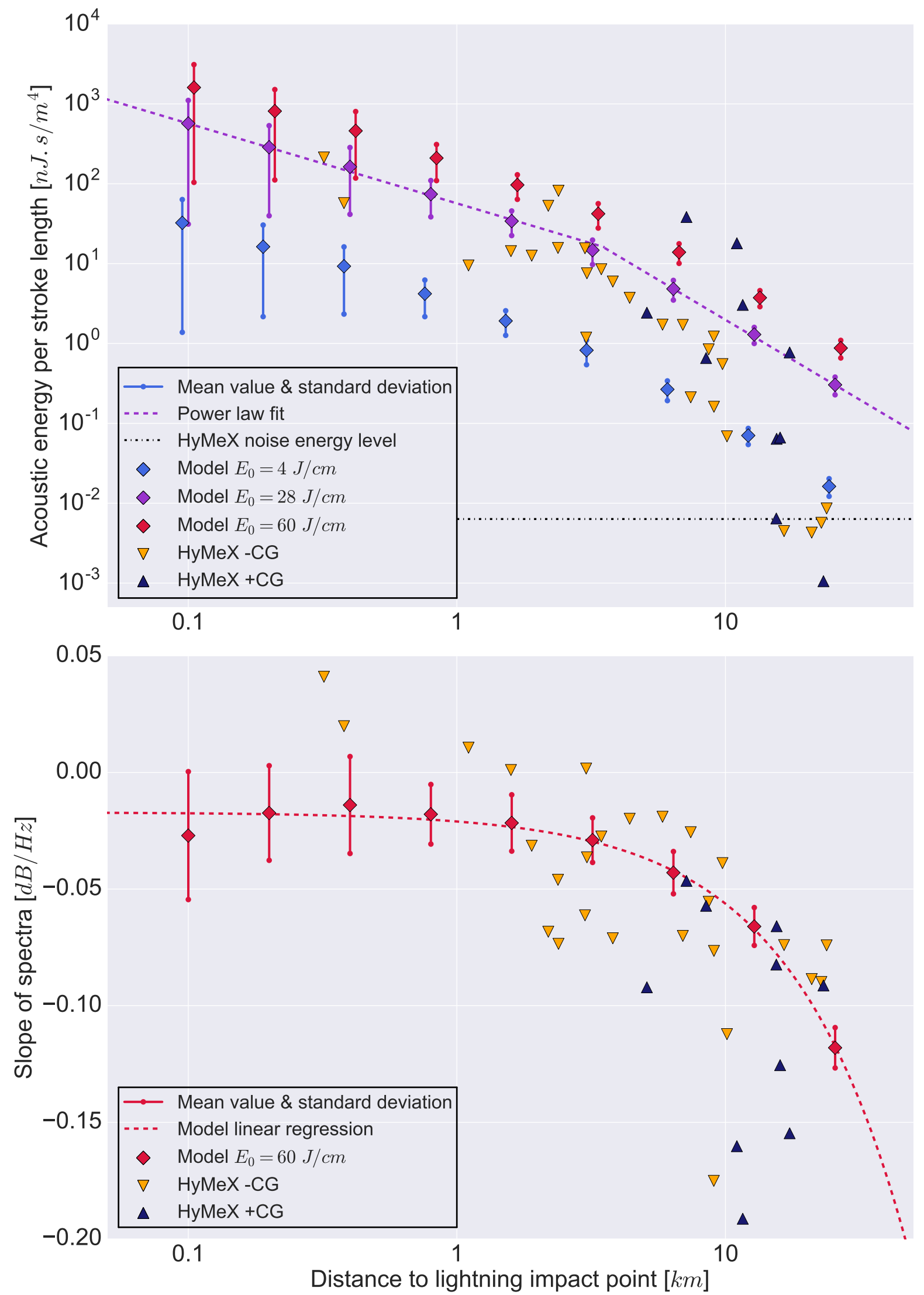

Figure 4. Statistical comparison between numerical results (diamonds: mean value, vertical step: standard deviation) and experimental data (triangles). (A) acoustic energy per stroke length and (B) slope of spectra linear regression versus distance to impact point. 